\title{
Explaining Resident Medical Students' Experiences of Performance During COVID-19: A Qualitative Study
}

\author{
Sara Esmaelzadeh (iD ${ }^{1}$, Fatemeh Aghamahdi (iD ${ }^{2}$, Roghayeh Mirmajidi ${ }^{3}$, Soraya Parvari ${ }^{4}$, Keshvad \\ Hedayatian $^{5}$ and Mohsen Arabi ${ }^{6, *}$ \\ ${ }^{1}$ Reproductive health Department, Social Determinants of Health Research Center, Alborz university of Medical Sciences, Karaj, Iran \\ ${ }^{2}$ Department of Pediatrics, Non-communicable Diseases Research Center, School of Medicine, Alborz University of Medical Sciences, Karaj, Iran \\ ${ }^{3}$ Department of Obstetrics and Gynecology, Kamali Hospital, School of Medicine, Alborz University of Medical Sciences, Karaj, Iran \\ ${ }^{4}$ Department of Anatomical Sciences, School of Medicine, Alborz University of Medical Sciences, karaj, Iran \\ ${ }^{5}$ Shahid Rajaei Hospital, School of Medicine, Alborz University of Medical Sciences, Karaj, Iran \\ ${ }^{6}$ Physiology, Pharmacology and Medical Physics Department, School of Medicine, Alborz University of Medical Sciences, Karaj, Iran \\ "Corresponding author: Physiology, Pharmacology and Medical Physics Department, School of Medicine, Alborz University of Medical Sciences, Karaj, Iran. Email: \\ samy.ghasemzadeh1394@gmail.com
}

Received 2021 August 21; Revised 2021 December 07; Accepted 2021 December 13.

\begin{abstract}
Background: The COVID-19 crisis has created many problems, including a negative impact on student education.

Objectives: The study aimed to understand the resident medical students' performance experiences during the COVID-19 pandemic, which is essential for future planning.

Methods: The present qualitative study was performed on 15 resident medical students in Alborz University of Medical Sciences, Iran, from September to November 2020. Data were analyzed using conventional content analysis.

Results: Analysis of the meaning units of interviews showed four categories describing the resident students' experiences of performance during COVID-19 as follows: Fear and anxiety, the need for support, the sense of responsibility, and the lack of educational facilities.

Conclusions: The present study showed that the COVID-19 pandemic had posed incredible new challenges for resident students. Assessing the mental health of residents, providing adequate protective equipment, and support from authorities and community members can create empathy, reduce the feeling of fear, and increase their accompaniment with pandemic managing programs. Due to the problems created in the training of residents during the pandemic, it seems necessary to propose rehabilitation courses to improve the training program for residents in various fields.
\end{abstract}

Keywords: Experiences, Residents, COVID-19, Content Analysis

\section{Background}

COVID-19 is now one of the main concerns of human society. It was first identified in Wuhan, China, in late 2019 and has infected almost every country globally (1). Coronaviruses affect the human respiratory system and induce severe respiratory disease (2). The COVID-19 virus is easily transmitted from person to person and has caused fear and anxiety behaviors in people worldwide (3). According to published statistics, COVID-19 has afflicted more than $2,300,000$ people in Iran (4). Fear of the COVID-19 outbreak has affected people's behavior and increased the virtual education system. Based on the proposed protocols, prevention methods include wearing a mask, hand washing, proper ventilation, observing social distance, and performing COVID-19 tests (5-7). During the COVID-19 outbreak, healthcare staff worked long hours and stayed away from the family for a long time; thus, mental disorders are more common in them, and we need to investigate their psychological issues and problems at work $(8,9)$. In addition to economic problems, the COVID-19 crisis has had a profound and unpredictable effect on education. For example, it has increased the virtual education system (10). The limited educational process of residents during the pandemic that requires personal distance, long periods of separation, and social commitment to avoid spreading the disease should be considered in the residents' educational program (11). Understanding the impact of COVID-19 on physicians working in hospitals creates strategies to support their mental health in such pandemics (12).

There are $75 \%$ of the students in eight fields of study at Alborz University of Medical Sciences. In the COVID-19 pan- 
demic, resident students in pediatrics, anesthesia, surgical, and internal medicine wards were also required to provide services in the COVID-19 intensive care units. Therefore, it is imperative to consider their experiences and feelings in the educational planning in this period. Therefore, since no qualitative study has been done in this regard, the current study was done to explain the experiences of medical students studying at COVID-19 intensive care units during the pandemic in Karaj, Alborz province, Iran.

\section{Methods}

The present qualitative study was performed on $15 \mathrm{Al}$ borz University of Medical Sciences students in Iran. The inclusion criteria included residents with experience of working in a COVID-19 intensive care unit. The exclusion criteria included having immune system problems and pregnancy.

\subsection{Ethical Consideration}

The Ethics Committee of Alborz University of Medical Sciences approved this study with code IR.ABZUMS.REC.1400.075. All participants were informed of the purposes and methods of this study. Informed consent of all respondents was an essential aspect of this research project, and those who agreed to participate in the study provided written consent.

\subsection{Data Collection}

We conducted in-depth, semi-structured interviews. Participants were selected by purposeful sampling with maximum variation. Initially, the first interview was conducted by the first and corresponding authors, and the subsequent interviews were conducted after the approval of other members of the research team. Interviews continued until data saturation was reached and the researcher no longer obtained new data. Data collection was done between September and November 2020 in Karaj, Iran. All students were interviewed in a private room in the medical university. Only the interviewer and the participant were present in the interview place. Interviews took 45 - $90 \mathrm{~min}$ on average.

An open question guide form was used in the semistructured interviews. Interview questions are in Box 1. These questions were only for the interview guide, and subsequent questions were asked based on the initial answers of the individuals. Each interview of participants was done only once.

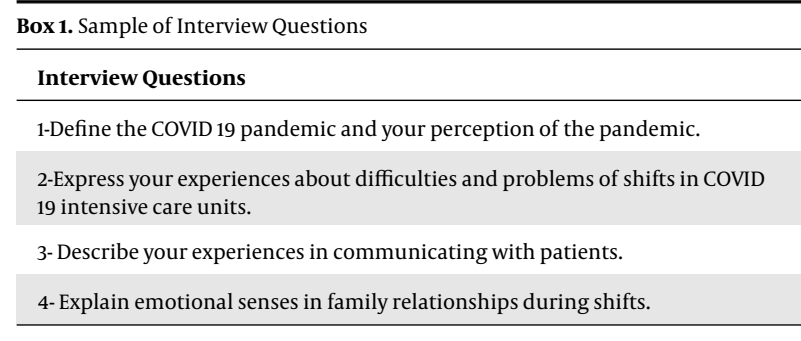

\subsection{Data Analysis}

Data were analyzed using conventional content analysis. Content analysis is a flexible method for analyzing text data. It is generally done when existing research literature on a phenomenon is limited. Data analysis was performed through the method of Graneheim and Lundman, 2004. The following steps were taken to analyze the collected data:

(1) Transcribing the interviews verbatim and reading them several times to get a general sense of the material.

(2) Dividing the text into meaning units, which are key phrases in the text.

(3) Abstracting the condensed meaning units and outlining with codes.

(4) Grouping the codes into subcategories and categories based on their similarities and differences (13).

\subsection{Rigor}

To ensure the accuracy and precision of the data, we used the Lincoln and Guba criteria, including credibility, dependability, conformability, and transferability (14). Credibility was established through member checking, peer checking, and prolonged engagement. Member checking was done by asking the respondents to approve the transcripts and emerging interview codes. Research teams consulted to deal with any ambiguities in the coding process, categories, and themes. In areas where the two researchers did not agree, definitions were clarified, and discussion continued until consensus was reached. The complete set of data analysis documents is on a file and available upon request for addressing transferability. For establishing dependability, external audits were used. We asked a researcher not involved in the research process to examine the process and product of the research for accuracy and evaluate whether or not the data supported the findings, interpretations, and conclusions. For establishing confirmability, the researcher documents the procedures for checking and rechecking the data during the entire research. These records contain what was done in the investigation and are available upon request. 


\section{Results}

Participants' demographic characteristics are mentioned in Table 1.

\begin{tabular}{|c|c|}
\hline Course & Frequency \\
\hline Internal medicine & 5 \\
\hline Pediatric & 4 \\
\hline General surgery & 2 \\
\hline Anesthesiology & 4 \\
\hline \multicolumn{2}{|l|}{ Gender } \\
\hline Female & 9 \\
\hline Male & 6 \\
\hline \multicolumn{2}{|l|}{ Marriage } \\
\hline Married & 10 \\
\hline Single & 5 \\
\hline \multicolumn{2}{|c|}{ Infection with COVID-19 } \\
\hline Yes & 6 \\
\hline No & 9 \\
\hline \multicolumn{2}{|l|}{ Age } \\
\hline Mean \pm SD & $36.7 \pm 7.02$ \\
\hline \multicolumn{2}{|l|}{ COVID-19 Shifts (mo) } \\
\hline Mean \pm SD & $5.5 \pm 2.1$ \\
\hline Min & 2 \\
\hline Max & 10 \\
\hline
\end{tabular}

In the study, 180 codes and four categories were extracted from interviews. The categories and subcategories are mentioned in Table 2. They included (1) Fear and anxiety: A) fear of the unknown, B) fear of transmitting the disease to others, and C) fear of COVID-19 stigma, (2) The need for support: A) support from family and B) support from administrators, (3) Sense of responsibility: A) professional commitment and B) patriotism, and (4) Lack of educational facilities: A) financial problems, B) lack of protective facilities and C) educational problems of students.

\subsection{Fear and Anxiety}

The results showed that most medical students experienced fear and anxiety, which eventually diminished and normalized. However, the prolongation of the pandemic caused fatigue in the resident students. One of the internal students, in the third year of the study, said: "In our opinion, COVID-19 was an unknown and deadly disease, and we were very stressed and afraid of getting sick. In the early days of the pandemic, we were constantly monitored for
COVID-19, but gradually our sensitivity reduced. At first, because of the new experiences, the difficult working conditions were tolerable. Now, because the incidence of the disease has multiplied and the pandemic has prolonged, the working conditions have become difficult for me." (Participant No. 3)

Another student said: "I contracted COVID-19 in March 2020. At that time, there was little information about the disease, and it was not very common. I had a fever and chills for several months and had a horrible experience. I was less scared at work because I got sick earlier than others." (Internal student, Participant No. 2)

\subsection{Fear of the Unknown}

COVID-19 is a new disease. There was no specific information about the disease and treatment protocols. Also, the fear of infection and the uncertainty of its consequences caused more fear and anxiety. One of the pediatric students who had to work in the COVID-19 ward said: "In April 2020, I was forced to work in the COVID-19 intensive care unit. At first, the nurses and we were very stressed and worried because the disease was unknown, and there was no specific treatment protocol. Nevertheless, now I have more information about the disease, and I know that following the protocols can prevent the disease." (Participant No. 5)

One of the anesthesia students said: "I was confused about how to treat the disease. I was confused about the patients' lungs, which became inflamed and deadly in just a few days." (Anesthesiology student, Participant No. 11)

\subsection{Fear of Transmitting the Disease to Others}

Fear of transmitting COVID-19 to their families and others was one of the students' fears that made it difficult for them to adapt to the new condition. One of the anesthesiology students who was married and had two children said: "I was worried about being a carrier of the disease and making my family members sick. So, I took my children to their grandparents' house and could not see them for a long time." (Participant No. 6).

Another married anesthesia student said: "I go home once a month, and when I get home, I take off my clothes and go to the bathroom. Also, my room is in an isolated house, and no one comes to it. In addition, COVID-19 prevented me from seeing my parents in another city." (Participant No. 7).

\subsection{Fear of COVID-19 Stigma}

One of the fears of students was the fear of disclosing COVID-19 infection to patients due to the stigma caused by the disease. The COVID-19 stigma that was the construct 


\begin{tabular}{|c|c|c|}
\hline Category & Subcategory & Codes \\
\hline \multirow{3}{*}{ Fear and anxiety } & Fear of the unknown & \multirow{3}{*}{$\begin{array}{l}\text { Fear of the COVID-19 infection; Concerns for the senior members of } \\
\text { the family; Fear of human factors on the spread of the disease }\end{array}$} \\
\hline & Fear of transmitting the disease to others & \\
\hline & Fear of COVID-19 stigma & \\
\hline \multirow{2}{*}{ The need for support } & Support from family & \multirow{2}{*}{$\begin{array}{l}\text { It was my family support that helped me continue my shifts; We } \\
\text { expected authorities to support us more }\end{array}$} \\
\hline & Support from administrators & \\
\hline \multirow{2}{*}{ Sense of responsibility } & Professional commitments & $\begin{array}{l}\text { Because I am a doctor, I considered it my duty to help with the } \\
\text { COVID-19 shifts. }\end{array}$ \\
\hline & Patriotism & I am proud of being helpful to my country \\
\hline \multirow{3}{*}{ Lack of educational facilities } & Financial problems & The salaries of residents did not change in the pandemic. \\
\hline & Lack of protective equipment & Shortage of protective equipment \\
\hline & Educational problems of students & Lack of a proper training team at the beginning of the pandemic \\
\hline
\end{tabular}

of community, because of isolation of patients, the rapid spread of disease, and death of the patients, increased problems in communication with patients and their relatives. One of the general surgery students said: "In the early days of the pandemic, people wanted to hear that their patient is not infected with COVID-19 virus due to psychological problems such as burying corpses of coronary patients with particular conditions and lack of mourning. It caused problems in communicating with patients (Participant No.12).

\subsection{The Need for Support}

Many students were from unrelated fields of study and had to work in the COVID-19 intensive care units. They considered these conditions mandatory and expected more support from university administrators. The disproportionate distribution of hospital working time in COVID-19 intensive care units was another reason for student dissatisfaction. Some students expressed the support of their families and relatives as a critical factor in continuing to work in the COVID-19 intensive care unit.

\subsection{Support from Administrators}

One of the pediatric students said: "We were unhappy and stressed because we had to work in the COVID-19 intensive care unit. Because working in this part of the hospital had no relation with our field of study, we expected more support and cooperation from university administrators." (Participant No. 6)

A pediatric student said: "University administrators promised to increase student salaries, but our salaries did not change. In addition, we had to buy our own protective equipment. Furthermore, the disproportionate distribution of hospital working time in the COVID-19 intensive care unit increases our protest." (Participant No. 8).

\subsection{Support from Family}

Some students mentioned that family support was a stimulator for continuous education and working during the pandemic. A pediatric student reported that: "My wife and family supported me psychologically, reducing my stress and making it better for me to work at the hospital." (Participant No. 4).

Another student declared that: "The support of my husband and his family enabled me to continue working in the hospital." (Participant No. 11).

\subsection{Sense of Responsibility}

Many students considered working in the COVID-19 intensive care unit to be a factor in self-improvement and declared that this condition is an opportunity for presenting their medical responsibility. Two students said: "We are proud to have the opportunity to help people in this critical situation." (Participants No. 1 and 4 )

\subsection{Patriotism}

Some students said that the sense of patriotism increased their hardworking in the hospital. A student said that: "Because of my strong sense of patriotism, I volunteered to work in a hospital. I was proud to help my country in this critical situation. I was also happy that other agencies and the media were psychologically supporting us." (Participant No. 5)

\subsection{Lack of Educational Facilities}

Some students were dissatisfied with many hospital hours, lack of protective facilities, and low salaries. 


\subsection{Financial Problems}

Some students declared that they bought protective equipment with their own money. An internal student explained: "The number of internal students was nine in total, which increased the number of working hours in the hospital. Also, our salaries were low and did not change with the increased working hours." (Participant No. 3).

\subsection{Lack of Protective Facilities}

The lack of protective equipment was the biggest problem for students. A surgery student declared that: "At first, we did not have proper protective equipment at the workplace, and other students and we had to buy it at our own expense, but now the situation is much better." (Participant No. 9)

An internal student stated, "The restroom in the hospital was not separated. Also, the distance between the restroom and the workplace was long. In addition, I had skin allergies to gloves, and it was difficult for me to wear them for a long time." (Participant No. 3)

\subsection{Educational Problems}

Another problem was the lack of a proper training team at the pandemic's beginning. Furthermore, knowledge of disease treatment and protocols was low. Also, the residents' education had been interrupted during the pandemic. A pediatric student declared that: "COVID-19 had a very negative effect on our education and reduced the number of pediatric patients in our hospital. The quality of education also decreased in other fields." (Participant No. 5).

\section{Discussion}

The COVID-19 pandemic changed the university education system and students' mental health and educational skills. In this study, most students felt fear and anxiety while working in the hospital. Most students were afraid of the viral disease and the possibility of transmission to others. A study conducted in the USA(2020) found that $50 \%$ of the students felt fear and anxiety about COVID-19 and its transmission to others (15). Also, according to studies conducted in China and Italy, students experienced fear and anxiety $(16,17)$. Another research conducted in Pakistan showed that $60.3 \%$ of physicians feared getting sick, and $79.7 \%$ were afraid of transmitting the disease to their families (18).

Other consequences of fear in students were communication problems with the patients and relatives, which due to the stigma caused by the disease, prevented them from accepting the disease, leading to the fear of assistants exposing the disease to the patients and their relatives. A study showed that stigma caused by COVID-19 disease threatened the survival of physicians and patients (19). Another study showed that people with COVID 19 infection stigmatization is raised because of community construction. Economic losses have risen because people are avoiding contact with patients. The stigmatization can lead to physical violence, social isolation, denial of employment, and stigmatization in most patients because they do not seek care services (20).

Another category found in this study was the need for support from family and administrators. Most assistants needed more support from the university officials because they were suddenly forced to attend the COVID-19 ward. Our study is in line with Urooj et al.' study reporting that most doctors expected the administrators to provide full support, protective equipment, planning, and logical distribution of resources to eliminate future uncertainty. Administrators and organizational leaders should provide clear messages that clinicians are valued, and managing the pandemic together is the goal (18).

The other category was the sense of responsibility. The educational challenges during the assistantship of a large number of coronary patients in each shift, fear of infection, lack of facilities and equipment of professional responsibility, and national sweat caused the presence of the assistants in coronary wards, which caused pride in them. A study reported burnout in medical staff in critical situations. Helping assistants feel better about their roles and giving them support would improve their performance and encourage them during the COVID-19 pandemic (21). Due to the prolongation of the COVID-19 pandemic, the relevant organizations must support the medical staff to manage this pandemic.

Another problem of the students in this study was the lack of facilities and equipment, which included the lack of protective equipment, financial problems, lack of independent resting places, and a far distance from the workplace to the resting place in the hospital. Other studies showed that $60 \%$ of students were afraid of contracting the disease due to the lack of access to protective equipment (22). In our study, many students complained of the lack of proper training by the authorities concerning the COVID-19 treatment methods and considered it necessary to create a suitable training team with the same protocols to deal with the disease during the pandemic. A study on surgical students showed that their skills were affected during the COVID-19 pandemic, and students were anxious about their education. Furthermore, many elective and non-emergency surgeries were also reduced (23).

One of the limitations of this study was the small number of residents studying at Alborz University of Medical 
Sciences. Due to the new construction of this college, residents of many fields are not trained, and the fields with residents have a minimal number of students. Thus, it is suggested that a similar study be conducted in more prominent universities with a more significant number of students. Another limitation was the study of the structure and equipment of the university, which due to the new construction of the university, had shortcomings in terms of physical space and the number of faculty members, affecting the experience of residents. Therefore, a similar study is recommended on residents in more prominent universities.

\subsection{Conclusions}

The present study results showed that the COVID-19 pandemic had posed incredible new challenges for resident students. Assessing the residents' mental health and providing adequate protective equipment and support from authorities and community members can create empathy, reduce fear, and increase their accompaniment in pandemic managing programs. Due to the problems created in the training of residents during the pandemic, it seems necessary to propose holding rehabilitation courses to improve residents' training programs in various fields.

\section{Acknowledgments}

The authors would like to thank the Kamali Research Center and Development.

\section{Footnotes}

Authors' Contribution: Mohsen Arabi and Sara Esmaelzadeh conceived and designed the experiments; Fatemeh Aghamahdi and Soraya Parvari performed the experiments; Mohsen Arabi and Sara Esmaelzadeh analyzed and interpreted the data; Mohsen Arabi and Roghayeh Mirmajidi contributed to reagents, materials, analysis tools, or data; Sara Esmaelzadeh, Fatemeh Aghamahdi, Roghayeh Mirmajidi, Soraya Parvari, and Mohsen Arabi wrote the paper.

Conflict of Interests: The authors declare no conflict of interest.

Ethical Approval: The Ethics Committee of Alborz University of Medical Sciences approved this study with code IR.ABZUMS.REC.1400.075.

Funding/Support: Alborz University of Medical Sciences supported this research.

Informed Consent: Informed consent from all respondents was an essential aspect of this research project, and those who agreed to participate in the study provided written consent.

\section{References}

1. Singhal T. A Review of Coronavirus Disease-2019 (COVID-19). Indian J Pediatr. 2020;87(4):281-6. doi: 10.1007/s12098-020-03263-6. [PubMed: 32166607]. [PubMed Central: PMC7090728].

2. Chan JF, Yuan S, Kok K, To KK, Chu H, Yang J, et al. A familial cluster of pneumonia associated with the 2019 novel coronavirus indicating person-to-person transmission: a study of a family cluster. Lancet. 2020;395(10223):514-23. doi: 10.1016/s0140-6736(20)30154-9.

3. Kim SW, Su KP. Using psychoneuroimmunity against COVID-19. Brain Behav Immun. 2020;87:4-5. doi: 10.1016/j.bbi.2020.03.025. [PubMed: 32234338]. [PubMed Central: PMC7194899].

4. Worldmeter. COVID-19 coronavirus pandemic February 18. 2021, [updated January 09, 2022]. Available from: https://www.worldometers. info/coronavirus/.

5. Daniel SJ. Education and the COVID-19 pandemic. Prospects (Paris). 2020:1-6. doi: 10.1007/s11125-020-09464-3. [PubMed: 32313309]. [PubMed Central: PMC7167396].

6. Huang Y, Zhao N. Mental health burden for the public affected by the COVID-19 outbreak in China: Who will be the high-risk group? Psychol Health Med.2021;26(1):23-34. doi:10.1080/13548506.2020.1754438. [PubMed: 32286091].

7. World Health Organization. Rational use of personal protective equipment for coronavirus disease (COVID-19): interim guidance. World Health Organization; 2020.

8. Tan W, Hao F, McIntyre RS, Jiang L, Jiang X, Zhang L, et al. Is returning to work during the COVID-19 pandemic stressful? A study on immediate mental health status and psychoneuroimmunity prevention measures of Chinese workforce. Brain Behav Immun. 2020;87:84-92. doi: 10.1016/j.bbi.2020.04.055. [PubMed: 32335200]. [PubMed Central: PMC7179503].

9. Zhang SX, Liu J, Afshar Jahanshahi A, Nawaser K, Yousefi A, Li J, et al. At the height of the storm: Healthcare staff's health conditions and job satisfaction and their associated predictors during the epidemic peak of COVID-19. Brain Behav Immun. 2020;87:144-6. doi: 10.1016/j.bbi.2020.05.010. [PubMed: 32387345]. [PubMed Central: PMC7199703].

10. Calhoun KE, Yale LA, Whipple ME, Allen SM, Wood DE, Tatum RP. The impact of COVID-19 on medical student surgical education: Implementing extreme pandemic response measures in a widely distributed surgical clerkship experience. Am J Surg. 2020;220(1):447. doi: 10.1016/j.amjsurg.2020.04.024. [PubMed: 32389331]. [PubMed Central: PMC7186124].

11. Hellewell J, Abbott S, Gimma A, Bosse NI, Jarvis CI, Russell TW, et al. Feasibility of controlling COVID-19 outbreaks by isolation of cases and contacts. Lancet Glob Health. 2020;8(4):e488-96. doi: 10.1016/s2214109x(20)30074-7.

12. Schwartz AM, Wilson JM, Boden SD, Moore TJ, Bradbury TJ, Fletcher ND. Managing Resident Workforce and Education During the COVID19 Pandemic: Evolving Strategies and Lessons Learned. JB JS Open Access. 2020;5(2). e0045. doi: 10.2106/JBJS.OA.20.00045. [PubMed: 33117955]. [PubMed Central: PMC7408274].

13. Graneheim UH, Lundman B. Qualitative content analysis in nursing research: concepts, procedures and measures to achieve trustworthiness. Nurse Educ Today. 2004;24(2):105-12. doi: 10.1016/j.nedt.2003.10.001. [PubMed:14769454].

14. Guba EG. Criteria for assessing the trustworthiness of naturalistic inquiries. Ectj.1981;29(2). doi: 10.1007/bf02766777.

15. Sanghavi PB, Au Yeung K, Sosa CE, Veesenmeyer AF, Limon JA, Vijayan V. Effect of the Coronavirus Disease 2019 (COVID-19) Pandemic on Pediatric Resident Well-Being. J Med Educ Curric Dev. 2020;7:2382120520947060. doi: 10.1177/2382120520947062. [PubMed: 32844117]. [PubMed Central: PMC7418226]. 
16. Wu Z, McGoogan JM. Characteristics of and Important Lessons From the Coronavirus Disease 2019 (COVID-19) Outbreak in China: Summary of a Report of 72314 Cases From the Chinese Center for Disease Control and Prevention. JAMA. 2020;323(13):1239-42. doi: 10.1001/jama.2020.2648. [PubMed: 32091533].

17. Slisco A. More than 50 doctors in Italy have now died from coronavirus. Newsweek. 2020.

18. Urooj U, Ansari A, Siraj A, Khan S, Tariq H. Expectations, Fears and Perceptions of doctors during Covid-19 Pandemic. Pak J Med Sci. 2020;36(COVID19-S4):S37-42. doi: 10.12669/pjms.36.COVID19-S4.2643. [PubMed: 32582312]. [PubMed Central: PMC7306962].

19. Bagcchi S. Stigma during the COVID-19 pandemic. Lancet Infect Dis. 2020;20(7). doi: 10.1016/s1473-3099(20)30498-9.

20. Prasad SK, Karahda A, Singh P, Gupta R. Role of mental health profes- sionals in dealing with the stigma attached to COVID-19. Gen Psychiatr. 2020;33(5). e100298. doi: 10.1136/gpsych-2020-100298. [PubMed: 32908960]. [PubMed Central: PMC7437609].

21. Roytman M, Shah S. Lessons learned during the COVID-19 pandemic: a single institution radiology chief resident experience. Clin Imaging. 2020;68:90-3. doi:10.1016/j.clinimag.2020.06.030. [PubMed: 32580107]. [PubMed Central: PMC7301115].

22. Bowden E, Campanile C, Golding B. Worker at NYC hospital where nurses wear trash bags as protection dies from coronavirus. New York Post. 2020.

23. Collins C, Mahuron K, Bongiovanni T, Lancaster E, Sosa JA, Wick E. Stress and the Surgical Resident in the COVID-19 Pandemic. J Surg Educ. 2021;78(2):422-30. doi: 10.1016/j.jsurg.2020.07.031. [PubMed: 32792326]. [PubMed Central: PMC7381910]. 